\title{
Improvement of free fatty acid production using a mutant acyl-CoA thioesterase I with high specific activity in Escherichia coli
}

\author{
Kwang Soo Shin ${ }^{1}$, Sangwoo Kim² and Sung Kuk Lee ${ }^{1,2^{*}}$
}

\begin{abstract}
Background: Microbial production of oleochemicals has been actively studied in the last decade. Free fatty acids (FFAs) could be converted into a variety of molecules such as industrial products, consumer products, and fuels. FFAs have been produced in metabolically engineered Escherichia coli cells expressing a signal sequence-deficient acyl-CoA thioesterase I ('TesA). Nonetheless, increasing the expression level of'TesA seems not to be an appropriate approach to scale up FFA production because a certain ratio of each component including fatty acid synthase and 'TesA is required for optimal production of FFAs. Thus, the catalytic activity of'TesA should be rationally engineered instead of merely increasing the enzyme expression level to enhance the production of FFAs.
\end{abstract}

Results: In this study, we constructed a sensing system with a fusion protein of tetracycline resistance protein and red fluorescent protein (RFP) under the control of a FadR-responsive promoter to select the desired mutants. Fatty acid-dependent growth and RFP expression allowed for selection of FFA-overproducing cells. A'TesA mutant that produces a twofold greater amount of FFAs was isolated from an error-prone PCR mutant library of E. coli'TesA. Its kinetic analysis revealed that substitution of $\mathrm{Arg}^{64}$ with $\mathrm{Cys}^{64}$ in the enzyme causes an approximately twofold increase in catalytic activity.

Conclusions: Because the expression of 'TesA in E. coli for the production of oleochemicals is almost an indispensable process, the proposed engineering approach has a potential to enhance the production of oleochemicals. The use of the catalytically active mutant 'TesA ${ }^{\mathrm{R} 64 \mathrm{C}}$ should accelerate the manufacture of FFA-derived chemicals and fuels.

Keywords: Error-prone PCR library, Escherichia coli, Free fatty acid, Oleochemicals, Thioesterase

\section{Background}

Among many molecules produced by microbes, fatty acids have many applications to the manufacture of biofuels, cosmetics, and pharmaceutical drugs [1-4]. There are two primary pathways for production of free fatty acids (FFAs): (1) phospholipid-derived FFAs, (2) microbial FFAs. The latter has several advantages: cost-effective production from renewable biomass, less production time, feasible extraction process, and direct conversion

\footnotetext{
*Correspondence: sklee@unist.ac.kr

${ }^{2}$ School of Energy and Chemical Engineering, Ulsan National Institute of Science and Technology (UNIST), Ulsan 44919, Republic of Korea Full list of author information is available at the end of the article
}

to oleochemicals [5-10]. These benefits of microbial production of FFAs have led to several studies aimed at increasing the FFA production through metabolic engineering and synthetic biology [11-14].

FFAs have been synthesized from acyl-ACPs-intermediates of iterative cycles of fatty acid synthetic pathway-by means of thioesterases. Among them, Escherichia coli acyl-CoA thioesterase I (TesA) has been frequently employed because its characteristics are well understood. TesA has $\mathrm{Ser}^{10}$-Asp ${ }^{154}$ - $\mathrm{His}^{157}$ as a catalytic triad and belongs to the family of SGNH-hydrolases [15]. The catalytic mechanism of the enzyme is as follows: the hydroxyl group of a serine residue nucleophilically 
attacks a thioester bond of the acyl-ACP or acyl-CoA with the assistance of the histidine residue [16]. TesA in a leaderless form ('TesA) has been used to produce FFAs in several studies $[6,12,17,18]$.

The optimal ratio of enzymes in a metabolic pathway is believed to be important for the improvement of product titers $[13,19]$. In the fatty acid synthetic pathway, nine distinct enzymes (FabA, B, D, F, G, H, I, Z, and ACP) interact with extended acyl-ACPs to synthesize longchain acyl-ACPs. An optimal molar ratio of the fatty acid synthase (FAS) components is the key for maximization of FAS activity. It was reported that the relative molar ratio of 'TesA to each FAS component is also required for maximal production of FFAs [18]. In addition, expression of 'TesA enhances FAS activity by hydrolyzing the longchain acyl-ACPs that inhibit several enzymes involved in the fatty acid synthesis [20]. According to these findings, overexpression of the wild type 'TesA may not be sufficient to maximize the production of FFAs in E. coli (Fig. 1a). We hypothesized that engineering 'TesA with a high specific activity is necessary for further improvement of the FFA production (Fig. 1b).

In this study, we constructed a mutant 'TesA library through error-prone PCR and developed a high-throughput screening (HTS) method to obtain FFA-overproducing strains. The catalytic properties of the desired mutant enzyme were characterized by biochemical assays. Finally, we demonstrated that the mutant 'TesA enzyme yielded a twofold greater amount of FFAs than the wild type enzyme did.

\section{Results and discussion}

Construction and characterization of a high-throughput screening system

Various sensing systems were previously employed to detect specific cellular signals and to improve production of valuable chemicals [14, 21, 22]. In this study, a fatty acid-sensing system was developed based on an existing system [11] to screen FFA-overproducing mutants in the 'TesA mutant library. We placed the TetA-RFP fusion protein rather than reporter RFP under the control of a FadR-regulated promoter, which comprised the $\mathrm{P}_{\mathrm{L}}$ promoter and FadR-binding sites. This screening system was expected to allow two selections: (1) FFA-dependent tetracycline resistance for enrichment of the expected mutants and (2) FFA-dependent RFP expression to identify the desired mutants and to eliminate false positive recombinants. FadR, an acyl-CoA-responsive transcriptional regulator, represses the expression of several genes prior to binding to long-chain acyl-CoAs [23]. In E. coli, acyl-CoAs can be synthesized from FFAs by acyl-CoA ligase (FadD). Thus, the intracellular concentration of FFAs and acyl-CoAs well correlate in E. coli [11]. At the low concentrations of FFAs or acyl-CoAs, FadR may consistently inhibit the expression of TetA-RFP in our sensing system. However, the high level of FFAs or acyl-CoAs should antagonize FadR and activate the expression of TetA-RFP. This sensing system was designated as the Fatty Acid Biosensor (FAB, Fig. 2a).

First, we confirmed a dose response of the FAB toward exogenous FFAs. When E. coli MG1655 harboring pFAB,

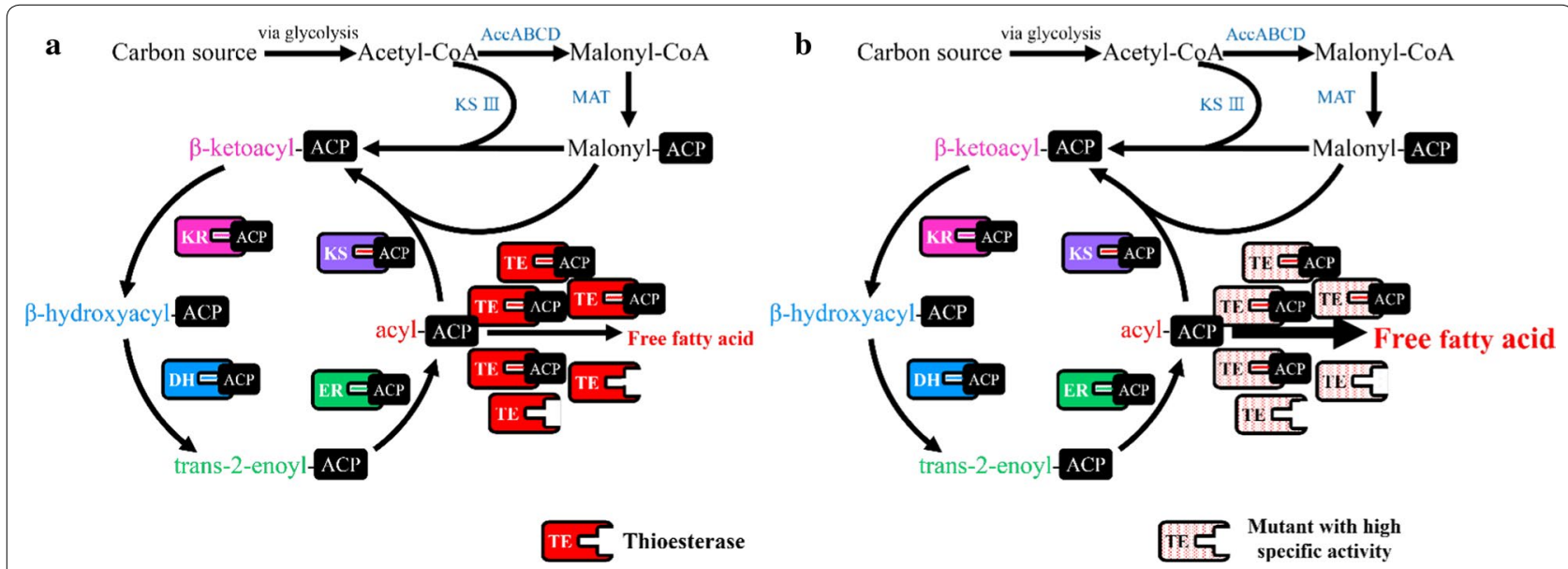

Fig. 1 A schematic diagram of fatty acid synthesis in E. coli. a FFA production is improved up to a certain protein level of a wild type thioesterase; however, it does not improve further even at its highest level because of stoichiometric protein-protein interactions involved in fatty acid synthesis. b FFA production is improved when a catalytic active thioesterase is devised. AcCABCD acetyl-CoA carboxylase, MAT malonyl-CoA:ACP transacylase, DH hydroxyacyl-ACP dehydratase, ER enoyl-ACP reductase, KR ketoacyl-ACP reductase, KS III ketoacyl-ACP synthase III, KS ketoacyl-ACP synthase I and II, TE thioesterase, ACP acyl carrier protein 


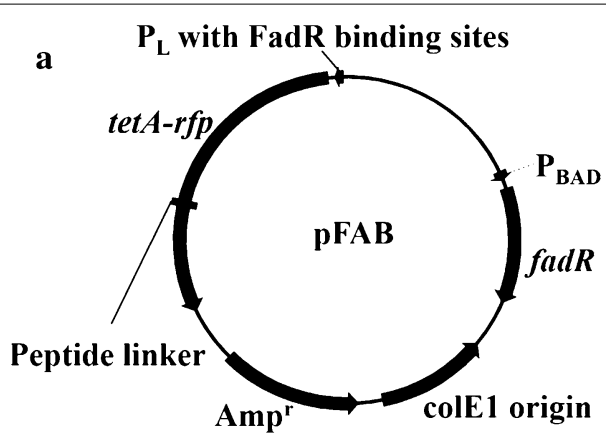

b

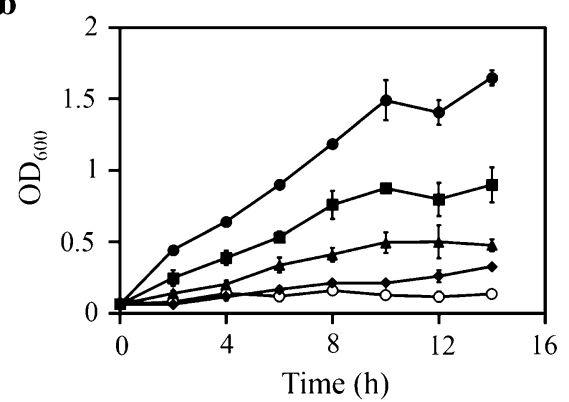

c

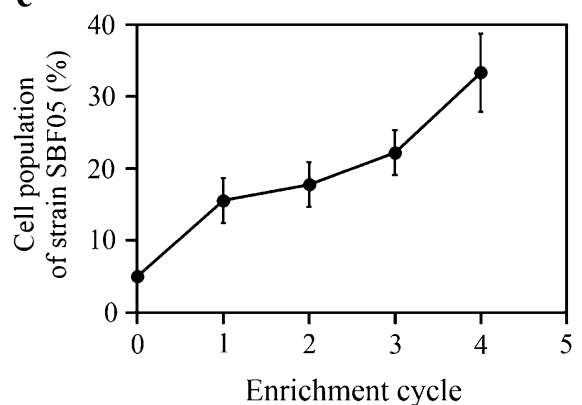

Fig. 2 Construction of the FFA-sensing plasmid and its performance. a The plasmid map of the fatty acid biosensor (pFAB); tetA: tetracycline resistance gene; $r f p$ : red fluorescent gene; Ampr: ampicillin resistance gene. $\mathbf{b}$ Effect of extracellular FFA concentrations on cell growth. At $40 \mu \mathrm{g} / \mathrm{mL}$ of tetracycline, SBF01 was cultivated with different concentrations of oleic acid (mM) [2 (filled circle), 1 (filled square), 0.5 (filled triangle), 0.1 (filled diamond), and 0 (open circle)]. c Screening efficiency. The strains SBF02 and SBF05 were cultivated in the 19:1 ratio to confirm that the FFA-overproducing cells were dominant in enrichment culture condition containing $40 \mu \mathrm{g} / \mathrm{mL}$ of tetracycline. The population of SBF05 was determined by colony PCR and DNA sequencing of the fadE locus of 15 randomly picked colonies after every enrichment cycle. Error bars mean standard deviations of three independent experiments

designated strain SBF01, was cultivated with various concentrations of oleic acid and $40 \mu \mathrm{g} / \mathrm{mL}$ of tetracycline, the fastest growth was observed in cells cultivated with the highest level of oleic acid tested (Fig. 2b). In addition, the RFP intensity of cells was well correlated with the growth of cells (Additional file 1: Figure S1), indicating that the sensing system could confer the corresponding tetracycline resistance and RFP intensity depending on the levels of FFAs. Furthermore, we measured the functionality of sensing system to internally produced FFAs. Cell growth was measured in E. coli strains MG1655 harboring pFAB (SBF01), MG1655 harboring pFAB and pBbB6c-tesA (SBF02), and MG1655 with fadE deletion harboring $\mathrm{pFAB}$ and $\mathrm{pBbB6c}$-'tesA (SBF05). When tetracycline $(40 \mu \mathrm{g} / \mathrm{mL})$ was added to exponentially growing cells, the SBF05 grew faster than other two strains (Additional file 1: Figure S2a). As expected, the SBF05 produced the highest level of FFAs, around $365 \mathrm{mg} / \mathrm{L}$, 1.4- and 6.1-fold higher than others (Additional file 1: Figure S2b). In addition, RFP intensity was also correlated with the cell growth and FFA production of each strain (Additional file 1: Figure S2c). The fastest growth and highest RFP intensity of the SBF05 should be resulted from its high FFA production. These results imply that the constructed sensing system shows a functional response to FFAs or acyl-CoAs.

Finally, we tested whether the sensing system could act as a screening tool for the enrichment of FFA-overproducing cells. The $f a d E$-undeleted strain (SBF02) and the fadE-deleted strain (SBF05) were mixed in the 19:1 ratio and incubated with $40 \mu \mathrm{g} / \mathrm{mL}$ of tetracycline. After four cycles of the enrichment, the population of the SBF05 considerably increased to 30 from $5 \%$ after four cycles of the enrichment (Fig. 2c). This increased population of the FFA-overproducing strain (SBF05) clearly showed that the constructed sensing system detected the level of FFAs and selectively supported the growth of strains synthesizing a large amount of FFAs.

The FFA-overproducing cells showed short doubling time even at a high concentration of tetracycline (Additional file 1: Figure S2d), resulting from the increase in the ratio of the FFA-overproducing strain after four cycles of the enrichment culture (Fig. 2c). In addition, the different levels of intracellular and extracellular fatty acids resulted in changes of tetracycline resistance and RFP expression. These results indicate that the constructed sensing system effectively confers the corresponding tetracycline resistance and red fluorescence in response to FFAs produced by the cells. This genetic sensing system was used to screen 'TesA mutants for those with high enzymatic activity that enables cells to produce more FFAs.

\section{Genotypic and phenotypic analysis of isolated 'TesA mutants}

Three recombinant cells [(MG1655 expressing a mutant 'TesA ${ }^{\mathrm{A} 120 \mathrm{D} / \mathrm{A} 171 \mathrm{~V}}$ (SBF07), 'TesA ${ }^{\mathrm{R} 64 \mathrm{C}}$ (SBF08), or 'TesA ${ }^{\mathrm{D} 74 \mathrm{G}}$ (SBF09)] produced more FFAs than MG1655 expressing a wild type 'TesA (SBF06) (Fig. 3). The SBF07 had a mutant 'TesA with two point mutations: A120D and A171V. It was difficult to explain how two alterations 


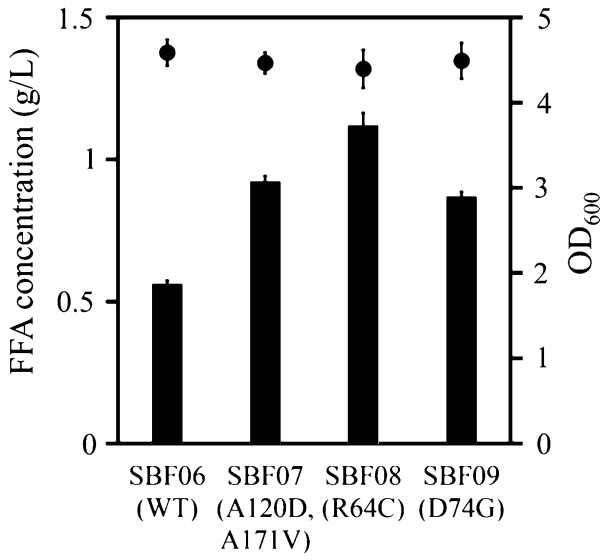

Fig. 3 Free fatty acid production of selected strains from the 'TesA mutant library. The expression of 'TesA was induced with $0.3 \mathrm{mM}$ IPTG, and the cells were cultivated for $48 \mathrm{~h}$ post-induction. Parenthesises indicate mutation of the 'TesA. Filled circles indicate the optical density of each culture after $48 \mathrm{~h}$. Error bars mean standard deviations of three independent experiments

improve the FFA production because there are no studies on the $\mathrm{Ala}^{171}$ residue. On the other hand, the $\mathrm{Ala}^{120}$ residue is a part of the loop (residues 111-120) located around a substrate-binding region. The loop slightly moves when 'TesA interacts with its substrate [24]. Thus, we can hypothesize that the mutation positively influences substrate-binding affinity and improves the FFA production. Strain SBF08, which expresses a mutant 'TesA with a substitution of longer and basic arginine with sulfur-containing cysteine at position 64 th, showed the highest production of FFAs $(\sim 1.1 \mathrm{~g} / \mathrm{L})$ among the three recombinants. This concentration was almost twofold higher than the amount produced by the SBF06. The SBF09 strain harboring 'TesA ${ }^{\mathrm{D} 74 \mathrm{G}}$ produced $\sim 1.5$-fold higher FFAs than the SBF06. As an N-terminus of the flexible loop (residues 75-80) in 'TesA, the $\mathrm{Asp}^{74}$ controls movements of the loop during substrate binding [24]. Thus, the alteration of $\mathrm{Asp}^{74}$ may increase the FFA production in a manner similar to that of the SBF07. The three mutants showed quite similar fatty acid distribution as compared with the SBF06 (Additional file 2: Table S1), indicating that the substitutions are not likely to significantly influence substrate specificity.

Alteration of enzyme characteristics such as substrate specificity [25], activity [26], and deregulated allosteric inhibition [27] has been used to increase production of desired products. The three mutants (strain SBF07, SBF08, and SBF09) isolated from the 'TesA mutant library accounted for $\sim 22,33$, and $22 \%$ of 18 selected mutants, respectively. This result indicates that the constructed sensing system selectively supported the growth of FFA-overproducing cells in the mutant library. The mutations in 'Tes $\mathrm{A}^{\mathrm{A} 120 \mathrm{D} / \mathrm{A} 171 \mathrm{~V}}$ and 'TesA $\mathrm{D}^{\mathrm{D} 4 \mathrm{G}}$ might cause a conformational change in the loop regions mentioned above and subsequently improve the substrate-binding affinity as previously reported [24], resulting in FFA overproduction. Given that no studies have shown the improvement of FFA production by altering amino acids in 'TesA to date, our results may offer target amino acid residues for the engineering of 'TesA.

\section{The effect of mutation at $\mathrm{Arg}^{64}$ of 'TesA on enzymatic activity}

In order to identify the substitution leading to the highest thioesterase activity, we performed site-directed mutagenesis on the $\mathrm{Arg}^{64}$ residue. Replacement of arginine with threonine ('TesA ${ }^{\mathrm{R} 64 \mathrm{~T}}$ ) or glutamine ('TesA ${ }^{\mathrm{R} 64 \mathrm{Q}}$ ) showed a 1.6- and 1.2-fold increase in the FFA production, respectively (Fig. 4). Among the cells isolated from the targeted random mutant library, a mutant with 'TesA ${ }^{\mathrm{R} 64 \mathrm{C}}$-like strain SBF08 produced the largest amount of FFAs (Fig. 4). This result indicates that this substitution might provide high catalytic activity although its location is far from an active site in the protein's structure [28]. In addition, the SBF08 showed comparable growth rate and glucose consumption rate with the SBF06 (Additional file 1: Figures S3a, b), but significantly higher FFA production (Additional file 1: Figure S3c), indicating that the expression of 'TesA ${ }^{\mathrm{R} 64 \mathrm{C}}$ could improve final titer, productivity, and yield.

Kinetic parameters of the 'TesA and 'TesA ${ }^{\mathrm{R} 64 \mathrm{C}}$ were analyzed in assays with palmitoyl-CoA to confirm the correlation between the increased FFA production level and increased enzymatic activity. As expected, $K_{\mathrm{m}}$ value

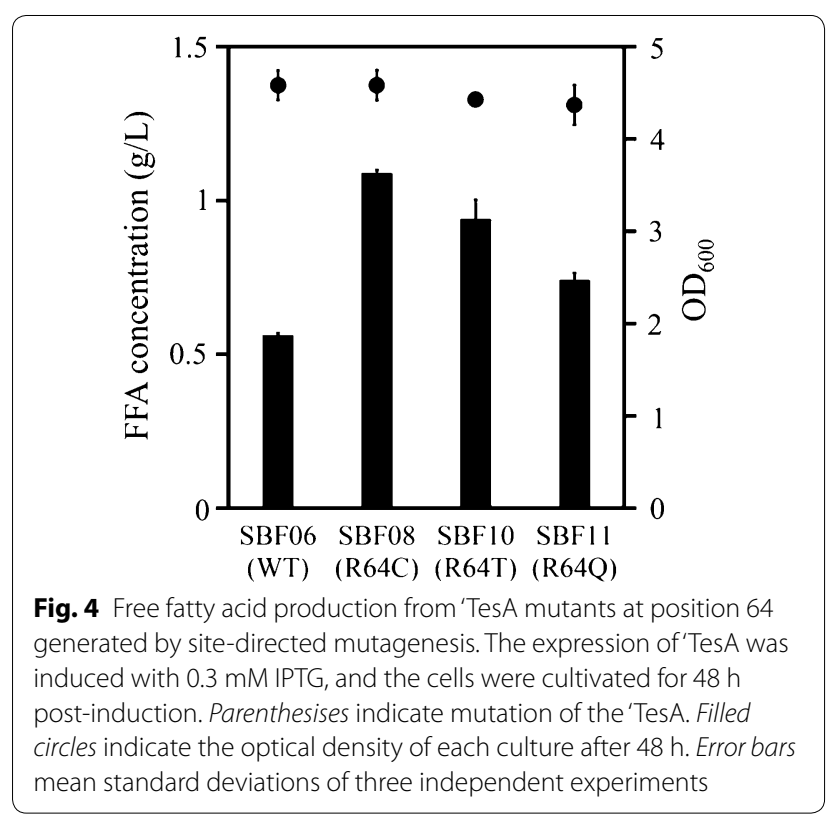


of the 'TesA ${ }^{\mathrm{R} 64 \mathrm{C}}$ toward palmitoyl-CoA was $\sim 60 \%$ of that of the 'TesA, indicating that the substitution increases the substrate affinity (Fig. 5). The $k_{\text {cat }}$ value of the engineered enzyme was $30 \%$ higher than that of the wild type enzyme, suggesting that the 'TesA ${ }^{\mathrm{R} 64 \mathrm{C}}$ more rapidly converts palmitoyl-CoA to palmitic acid. Therefore, these kinetic parameters can be considered supporting evidence of the highest amount of FFA synthesis in the SBF08.

Several residues of 'TesA have been studied to understand its kinetic behavior. The movement of the switch loop (residues 75-80) influences substrate specificity by stabilizing the Michaelis complex (MC) when 'TesA interacts with its substrate [24]. Moreover, mutation of $\operatorname{Trp}^{23}$ (not in the active site) significantly reduces the catalytic activity [29]. The promise of 'TesA engineering was previously seen in the 'TesA ${ }^{\mathrm{L} 109 \mathrm{P}}$, which shows altered substrate specificity [24]. Its use in engineered $E$. coli increased a proportion of short-chain FFAs, but not a total amount of FFAs [30]. It was previously reported that 'TesA and its substrate rapidly form MC; however, conversion into the tetrahedral complex is slow, suggesting that 'TesA is not likely to efficiently synthesize FFAs from acyl-ACPs [31]. Nevertheless, none of mutations in 'TesA were found to increase the production of FFAs to date. The 'TesA ${ }^{\mathrm{R} 64 \mathrm{C}}$ has higher substrate affinity and reaction rate than the wild type 'TesA, indicating that the increased specific activity of 'TesA improves the FFA production in E. coli as we hypothesized.

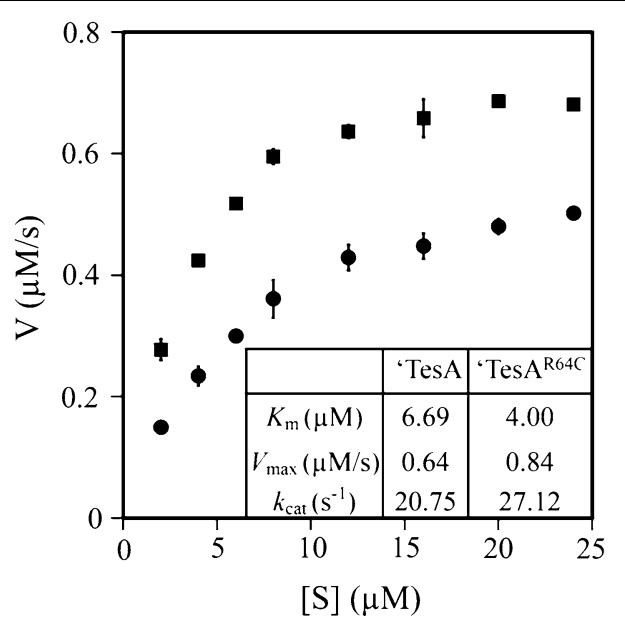

Fig. 5 Kinetic analysis of the 'TesA (filled circle) and 'TesA ${ }^{\text {R64C (filled }}$ square). The values are average of three independent experiments. The kinetic parameters were calculated by nonlinear repression plots of the Michaelis-Menten equation. The enzyme concentration was $3.1 \times 10^{-5} \mathrm{mM}$

\section{Increased FFA production driven by high specific activity} of 'TesA ${ }^{\text {R64C }}$

Multiple enzymatic activities are required to synthesize FFAs from acetyl-CoAs. It has been reported that the relative ratios of protein abundance of the FAS components are crucial for generating maximum synergy for the fatty acid synthesis [18]. Based on this fact, we hypothesized that high production of fatty acids can be achieved by optimal expression of 'TesA with high specific activity but not by overexpression of the wild type 'TesA. Fatty acid production was compared between cells expressing the wild type 'TesA or the catalytically active mutant 'TesA ${ }^{\mathrm{R} 64 \mathrm{C}}$ at different protein expression levels. As expected, there were slight increases in FFA production in both the strains, when they were induced with more than 0.125 mM IPTG (Fig. 6a). On the other hand, relative intensity values of each protein band were increased

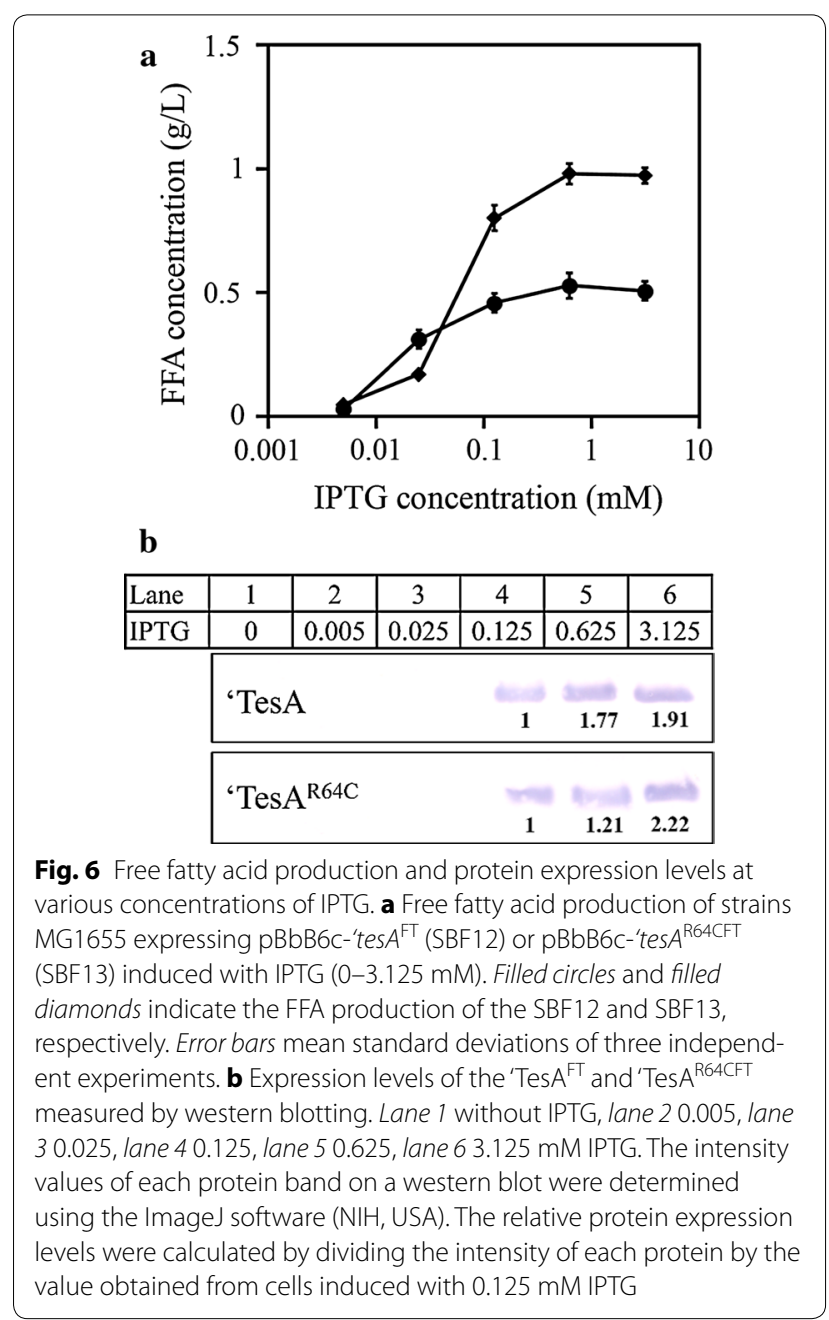


up to twofold in both the strains (Fig. 6b). The increase in protein levels in both the strains did not correspond to an increment in their FFA production, indicating that the thioesterase level produced even at $0.125 \mathrm{mM}$ IPTG may yield the optimal ratio for the maximal FAS activity. This result also supports previous report [18] that a certain stoichiometry of FAS protein components is necessary for maximal production of fatty acids in E. coli.

In other studies, the high concentration of acyl-ACPs has been reported to cause feedback inhibition of activities of three enzymes including ACC, FabH, and FabI [32, 33]. It also alters activity of FabF and FabB: conversion of malonyl-ACP into acetyl-ACP, subsequently transformed to acetyl-CoA by an action of FabH [34]. The other substrate of 'TesA, acyl-CoA, inhibits activity of FabI, which plays a crucial role in the fatty acid elongation [35]. These results indicate that the acyl-ACP and acyl-CoA are key regulatory signals in the fatty acid synthesis. Thus, the use of the catalytically active 'TesA ${ }^{\mathrm{R} 64 \mathrm{C}}$ might accelerate the production of FFAs and their derivatives with rapid conversion of acyl-ACPs or acyl-CoAs into FFAs. This finding supported our hypothesis that the way to increase FFA production is not the overexpression of 'TesA but the expression of more catalytically active 'TesA.

\section{Conclusions}

A certain ratio of 'TesA to FAS components during FFA synthesis is necessary for efficient interaction with its substrate, acyl-ACPs, and feedback inhibition of FAS enzymes is reduced by its activity. In order to increase FFA production, the expression of a catalytically active thioesterase rather than merely excessive protein expression was tested here as a key manipulation in E. coli. Catalytically active mutant thioesterases from a 'TesA mutant library were selected using tetracycline resistance marker for primary screening and RFP for secondary screening. The isolated 'TesA ${ }^{\mathrm{R} 64 \mathrm{C}}$ mutant yielded approximately twofold higher FFA production and catalytic activity as compared with the wild type 'TesA. The use of 'TesA ${ }^{\mathrm{R} 64 \mathrm{C}}$ would boost the production of fatty acid-based chemicals and fuels because FFAs are the precious precursors for their production.

\section{Methods}

\section{Bacterial strains and plasmids}

Strains, plasmids, and primers used in this study are listed in Table 1 and Additional file 2: Table S2. E. coli MG1655 served as a parental strain for the construction of all the strains used in this study. Deletion of chromosomal fadE (acyl-CoA dehydrogenase gene) was performed by means of $\lambda$-recombination system as described elsewhere [36].

To generate all the plasmids harboring 'tes $A$ variants, the BioBrick plasmid pBbB6c- $g f p$ served as a starting vector, and we replaced the $g f p$ insert with PCR-amplified 'tes $A$ alleles after digestion of the plasmid and PCR products with EcoRI and XhoI. The tes $A$ and tes $A^{\mathrm{R} 64 \mathrm{C}}$ alleles were also cloned into the NcoI- and XhoI-digested pET28a expression vector for purification of His-tagged proteins. The biosensor plasmid pFAB was constructed by cloning $E$. coli fadR and a tetA-rfp fusion gene with a FadR-responsive promoter into pBbE8a-rfp. In brief, $f a d R$ of $E$. coli MG1655 was amplified by means of primers, FadR FP and FadR RP, and ligated into pBbE8a-rfp after digestion with $B g l \mathrm{II}$ and $\mathrm{XhoI}$, resulting in the pBbE8a$f a d R$ vector. The translational fusion of tet $A$ and $r f p$ linked by a flexible peptide linker with GGGS $\times 4$ [37] was ligated with $\mathrm{P}_{\mathrm{L}}$ promoter and FadR-binding sites [11] using splice overlap extension (SOE)-PCR [38], and the product was cloned into the AatII-digested pBbE8a-fadR vector.

\section{Media and cultivation conditions}

The response of the fatty acid-sensing system to fatty acids was measured as previously described [23]. An overnight culture grown in rich broth (RB) (10 g of tryptone, $5 \mathrm{~g}$ of $\mathrm{NaCl}$ and $1 \mathrm{~g}$ of yeast extract per liter) was inoculated at 1:100 into $5 \mathrm{~mL}$ of the fresh $\mathrm{RB}$ medium supplemented with the various concentrations of oleic acid $(0,0.1,0.5,1$, or $2 \mathrm{mM})$ and $40 \mu \mathrm{g} / \mathrm{mL}$ of tetracycline. Tween 20 was added to final concentration of $0.5 \%$ to solubilize the oleic acid. After $24 \mathrm{~h}$ of growth at $37^{\circ} \mathrm{C}$, RFP fluorescence and optical density at $600 \mathrm{~nm}\left(\mathrm{OD}_{600}\right)$ were measured on a 96-well microplate reader (Infinite M200 TECAN, Austria) and a spectrophotometer (Libra S22, England). The fluorescence was then normalized to $\mathrm{OD}_{600}$ (RFP fluorescence per $\mathrm{OD}_{600}$ unit). The response of sensing system to internally produced FFAs was measured as cultivating various strains in the M9 medium supplemented with $2 \mathrm{mM} \mathrm{MgSO}_{4}, 0.1 \mathrm{mM}$ of $\mathrm{CaCl}_{2}$, additional $100 \mathrm{mM}$ of sodium phosphate buffer ( $\mathrm{pH} 7.0)$, and $0.6 \%$ of glycerol. Addition of $0.3 \mathrm{mM}$ IPTG and $40 \mu \mathrm{g} / \mathrm{mL}$ of tetracycline was conducted at $\mathrm{OD}_{600}$ of 0.5 . Each parameter such as $\mathrm{OD}_{600}$, RFP intensity, and FFA concentration was measured as mentioned above and 'Analysis of FFA and glucose' section, respectively.

To analyze the FFA production, a single colony was picked from an agar plate and incubated with $5 \mathrm{~mL}$ of a LB medium at $37^{\circ} \mathrm{C}$. An overnight culture was washed with distilled water twice and inoculated at the inoculum size of $3 \%$ into $5 \mathrm{~mL}$ of the M9 medium supplemented with $2 \mathrm{mM} \mathrm{MgSO}_{4}, 0.1 \mathrm{mM} \mathrm{CaCl}{ }_{2}$, additional $100 \mathrm{mM}$ sodium phosphate buffer ( $\mathrm{pH} 7.0$ ), and $2 \%$ of glucose. The cells were cultivated at $30{ }^{\circ} \mathrm{C}$ for $48 \mathrm{~h}$. The expression of thioesterases was induced by the addition of $0.3 \mathrm{mM}$ IPTG at $\mathrm{OD}_{600}$ of 0.5 . To measure several parameters such as cell density, glucose concentration, and 
Table 1 Strains and plasmids used in this study

\begin{tabular}{|c|c|c|}
\hline Strains and plasmids & Genotype and description & Source \\
\hline \multicolumn{3}{|l|}{ Strains } \\
\hline MG1655 & E. coli K-12 $\mathrm{F}^{-} \lambda^{-} i / v G^{-} r f b-50 r p h-1$ & {$[40]$} \\
\hline SBF01 & MG1655 with pFAB & This study \\
\hline SBF02 & MG1655 containing pFAB and pBbB6c-'tesA & This study \\
\hline SBF03 & MG1655 with $\triangle$ fadE::FRT & This study \\
\hline SBF04 & MG1655 with $\triangle$ fadE::FRT containing pFAB & This study \\
\hline SBF05 & MG1655 with $\triangle$ fadE::FRT containing pFAB and pBbB6c-'tesA & This study \\
\hline SBF06 & MG1655 with pBbB6c-'tesA & This study \\
\hline SBF07 & MG1655 with pBbB6c-'tesA A120D, A171V & This study \\
\hline SBF08 & MG1655 with pBbB6c-'tes $A^{\text {R64C }}$ & This study \\
\hline SBF09 & MG1655 with pBbB6c-'tesA $A^{\mathrm{D} 74 \mathrm{G}}$ & This study \\
\hline SBF10 & MG1655 with pBbB6c-'tesA $A^{\text {R64T }}$ & This study \\
\hline SBF11 & MG1655 with pBbB6c-'tesA ${ }^{\text {R64Q }}$ & This study \\
\hline SBF12 & MG1655 with pBbB6c-'tesA $A^{\mathrm{FT}}$ & This study \\
\hline SBF13 & MG1655 with pBbB6c-'tesA R64CFT $^{\text {Re }}$ & This study \\
\hline SBL01 & MG1655 library containing pFAB and mutated 'TesA & This study \\
\hline BL21(DE3) & $\begin{array}{l}\text { E. coli B F-ompT gal dcm lon hsdS }{ }_{B}\left(r_{B}^{-} m_{B}^{-}\right) \lambda(\text { DE3 acl lacUV5-T7 } \\
\text { gene } 1 \text { ind1 sam7 nin 5]) }\end{array}$ & [41] \\
\hline BL21(DE3)-'TesA & BL21(DE3) with pET28a-'tesA & This study \\
\hline BL21(DE3)-'TesA ${ }^{R 64 C}$ & BL21(DE3) with pET28a-'tes $A^{R 64 C}$ & This study \\
\hline \multicolumn{3}{|l|}{ Plasmids } \\
\hline pBbE8a-rfp & ColE1 ori, carrying $\mathrm{P}_{\mathrm{BAD}}$ promoter and $r f p, \mathrm{Amp}^{\mathrm{R}}$ & {$[42]$} \\
\hline pBbE8a-fadR & pBbE8a with $\triangle r f p:: f a d R$ (from MG1655), Amp ${ }^{R}$ & This study \\
\hline pBBR1 MCS-3 & Cloning vactor carrying MCS and lacZ alpha, $\mathrm{TC}^{\mathrm{R}}$ & [43] \\
\hline $\mathrm{pFAB}$ & pBbE8a-fadR carrying $\mathrm{P}_{\mathrm{LR}}$-tetA-rfp on Aatll site & This study \\
\hline pBbB6c-gfp & BBR1 ori, carrying $\mathrm{P}_{\mathrm{L}-\mathrm{lac} 01}$ promoter and $g f p, \mathrm{Cm}^{\mathrm{R}}$ & {$[42]$} \\
\hline pBbB6c-tesA & pBbB6c-gfp with $\triangle g f p::$ tes $A, C^{R}$ & This study \\
\hline pBbB6c-'tes A $A^{A 120 D, ~ A 171 V ~}$ & pBbB6c-gfp with $\triangle g f p::{ }^{\prime}$ tesA $A^{A 120 D,}$ A171V, $\mathrm{Cm}^{R}$ & This study \\
\hline pBbB6c-tes $A^{\mathrm{R} 64 \mathrm{C}}$ & pBbB6c-gfp with $\triangle g f p::$ tesA $^{\mathrm{R} 64 \mathrm{C}}, \mathrm{Cm}^{\mathrm{R}}$ & This study \\
\hline $\mathrm{pBbB6c-'tes} A^{\mathrm{D} 74 \mathrm{G}}$ & pBbB6c-gfp with $\triangle g f p$ ::'tes $A^{\mathrm{D} 74 \mathrm{G}}, \mathrm{Cm}^{\mathrm{R}}$ & This study \\
\hline $\mathrm{pBbB6c}$-'tes $^{\mathrm{R} 64 \mathrm{~T}}$ & pBbB6c-gfp with $\triangle g f p:$. 'tes $A^{\mathrm{R} 64 \mathrm{~T}}, \mathrm{Cm}^{\mathrm{R}}$ & This study \\
\hline pBbB6c-'tes $A^{\text {R64Q }}$ & pBbB6c-gfp with $\triangle g f p$ ::'tes $A^{\mathrm{R} 64 \mathrm{Q}}, \mathrm{Cm}^{\mathrm{R}}$ & This study \\
\hline $\mathrm{pBbB6c-}{ }^{\prime} \operatorname{tes} A^{\mathrm{FT}}$ & pBbB6c- gfp with $\triangle g f p:$ :flag tagged'tes $A, \mathrm{Cm}^{\mathrm{R}}$ & This study \\
\hline pBbB6c-'tes $A^{\text {R64CFT }}$ & pBbB6c- gfp with $\triangle g f p::$ flag tagged'tes $A^{R 64 C}, \mathrm{Cm}^{R}$ & This study \\
\hline pET28a $(+)$ & Expression vector with $(\mathrm{His})_{6}$-tag, $\mathrm{Km}^{\mathrm{R}}$ & Novagen \\
\hline pET28a-'tesA & pET28a with C-terminally (His) 6 -tagged 'tesA & This study \\
\hline pET28a-'tesA $A^{\text {R64C }}$ & pET28a with C-terminally (His) ${ }_{6}$-tagged 'tes $A^{\mathrm{R} 64 \mathrm{C}}$ & This study \\
\hline
\end{tabular}

FFA concentration in the SBF06 and SBF08, cells were cultivated in mini-bioreactors as previously described [10]. Briefly, cells were grown in LB medium, which was used to inoculate 1:100 into $5 \mathrm{~mL}$ of the M9 medium supplemented with $2 \mathrm{mM}$ of $\mathrm{MgSO}_{4}, 0.1 \mathrm{mM}$ of $\mathrm{CaCl}_{2}$, $3 \%$ of glucose, $1 \mathrm{~g} / \mathrm{L}$ yeast extract, and trace elements. The trace elements consist of $2.4 \mathrm{~g}$ of $\mathrm{FeCl}_{3} \cdot 6 \mathrm{H}_{2} \mathrm{O}, 0.3 \mathrm{~g}$ of $\mathrm{CoCl}_{2} \cdot 6 \mathrm{H}_{2} \mathrm{O}, 0.15 \mathrm{~g}$ of $\mathrm{CuCl}_{2} \cdot 2 \mathrm{H}_{2} \mathrm{O}, 0.3 \mathrm{~g}$ of $\mathrm{ZnCl}_{2}$, $0.3 \mathrm{~g}$ of $\mathrm{Na}_{2} \mathrm{MO}_{4} \cdot 2 \mathrm{H}_{2} \mathrm{O}, 0.075 \mathrm{~g}$ of $\mathrm{H}_{3} \mathrm{BO}_{3}$, and $0.495 \mathrm{~g}$ of $\mathrm{MnCl}_{2} \cdot 4 \mathrm{H}_{2} \mathrm{O}$ per liter. Overnight grown cells in the minimal medium were harvested and resuspended in $70 \mathrm{~mL}$ of the fresh M9 medium. The $\mathrm{pH}$ was maintained at 7.0 with $2 \mathrm{~N} \mathrm{NaOH}$. IPTG $(0.3 \mathrm{mM})$ was added to induce the expression of thioesterase at $\mathrm{OD}_{600}$ of 1 .

\section{Mutant library construction}

Error-prone PCR mutagenesis of 'tesA was conducted, and the PCR products were cloned into EcoRI and XhoI sites of pBbB6c- $g f p$ by the Mutagenex Inc (http://www. mutagenex.com). The mutant library containing $10^{6}$ independent colonies was constructed without hot spots of mutations. 
Site-directed mutagenesis was performed by PCR amplification using the overlapping oligonucleotide primers containing degenerate codon NNS [39] to randomly replace the arginine at position 64 of 'TesA with another amino acid. Briefly, two overlapping PCR products were generated from tesA by means of the primer sets (TesA FP, TesA SD RP, TesA SD FP, and TesA RP). Overlapping PCR products were then spliced using the appropriate primer set (TesA FP and TesA RP). The final constructs were obtained by the same procedure that was used for plasmid construction. In total, 1016 colonies were generated.

\section{Enrichment and isolation of 'TesA mutants with increased activity}

To confirm the enrichment efficiency, two strains in a 19:1 ratio (SBF02-SBF05) were mixed and incubated in the LB medium supplemented with $0.5 \%$ of glucose, $0.1 \mathrm{mM}$ of IPTG, and $40 \mu \mathrm{g} / \mathrm{mL}$ of tetracycline. The cells were subcultured at 1:50 into the fresh medium every $24 \mathrm{~h}$. After each round of enrichment, cells from each mixed culture were plated on an agar plate and incubated at $37{ }^{\circ} \mathrm{C}$ overnight. Fifteen of the resulting colonies were randomly picked and subjected to colony PCR and DNA sequencing of the amplified $f a d E$ locus using primers ( $f a d E$ seq FP and $f a d E$ seq RP) to evaluate the proportion of SBF05 in each mixed population. For enrichment of FFA-overproducing cells, the 'TesA mutant library was transformed into strain SBF01, and $7.2 \times 10^{9}$ cells harboring mutant 'TesA were obtained. Three successive subcultures were performed by transferring $2 \%(\mathrm{v} / \mathrm{v})$ of the culture to the new subculture at 24-h intervals. The concentration of tetracycline was increased at every transfer $(40,50$, and $60 \mu \mathrm{g} / \mathrm{mL})$.

Cells enriched with tetracycline were sorted based on the RFP signal using a FACS Calibur instrument (BD Bioscience, USA). The sorted cells were recovered in $50 \mathrm{~mL}$ of the SOC medium at $37{ }^{\circ} \mathrm{C}$ for $1 \mathrm{~h}$ prior to plating on an agar plate. For single-cell culture analysis, colonies grown on the agar plate were cultivated in a deep 96-well plate with $200 \mu \mathrm{L}$ of the $\mathrm{LB}$ medium at $30{ }^{\circ} \mathrm{C}$ for $16 \mathrm{~h}$. Overnight cultures were transferred at 1:100 into a new deep 96-well plate filled with $400 \mu \mathrm{L}$ of the LB medium supplemented with $0.5 \%$ of glucose and $0.1 \mathrm{mM}$ IPTG. After $24 \mathrm{~h}$ of cultivation, the RFP signal was measured as described above.

\section{Analysis of FFA and glucose}

The concentration of FFAs was measured as previously described [6]. In a $2.0-\mathrm{mL}$ tube, $500 \mu \mathrm{L}$ of cultures was taken and acidified by the addition of $50 \mu \mathrm{L}$ of $6 \mathrm{~N} \mathrm{HCl}$. The FFAs were extracted by vigorous vortexing for $30 \mathrm{~s}$ with $500 \mu \mathrm{L}$ of ethyl acetate and $50 \mu \mathrm{L}$ of $1 \mathrm{~g} / \mathrm{L}$ methyl nonadecanoate (Sigma-Aldrich) as an internal standard. The organic layer was separated after centrifugation for $2 \mathrm{~min}$, and the same procedure was repeated with additional $500 \mu \mathrm{L}$ of ethyl acetate. Extracted FFAs were subjected to methylation with $100 \mu \mathrm{L}$ of the mixture $\mathrm{MeOH}: 6 \mathrm{~N} \mathrm{HCl}(9: 1, \mathrm{v} / \mathrm{v})$ and $100 \mu \mathrm{L}$ of TMS-diazomethane (Sigma-Aldrich). After incubation for 10-15 min, fatty acid methyl esters (FAMEs) were analyzed using a gas chromatograph (Agilent 7890A) equipped with a flame ionization detector (FID) and a DB-5 column (30 m $\times 0.25 \mathrm{~mm}$, Agilent Technologies) The identification and concentration of FAMEs were analyzed by comparing peaks with the external standards composed of C10-22 FAMEs (Sigma-Aldrich). Oven temperature of $60{ }^{\circ} \mathrm{C}$ was held for $2.5 \mathrm{~min}$ and ramped to $250{ }^{\circ} \mathrm{C}$ at $20^{\circ} \mathrm{C} / \mathrm{min}$, and then held for $4 \mathrm{~min}$. Finally, temperature reached $325{ }^{\circ} \mathrm{C}$ at $10{ }^{\circ} \mathrm{C} / \mathrm{min}$ and was held for $5 \mathrm{~min}$.

Residual glucose was analyzed using a Shimadzu HPLC station equipped with a refractive index detector (Shimadzu) and a SIL-20A auto-sampler (Shimadzu) as previously described [10]. Supernatant of each culture was collected and heated at $80{ }^{\circ} \mathrm{C}$ for $1 \mathrm{~h}$ prior to second centrifugation for $30 \mathrm{~min}$. The final samples were injected into a HPX-87P column (Bio-Rad) at $0.6 \mathrm{~mL} / \mathrm{min}$ with HPLC-grade water.

\section{Enzyme expression and kinetic analysis}

BL21(DE3) cells harboring pET28a-tes $A$ and pET28a'tes $A^{\mathrm{R} 64 \mathrm{C}}$ were cultured in the $\mathrm{LB}$ medium at $37{ }^{\circ} \mathrm{C}$ until $\mathrm{OD}_{600}$ reached 0.6. The expressions of 'TesA and 'TesA ${ }^{\mathrm{R} 64 \mathrm{C}}$ were induced by the addition of $0.1 \mathrm{mM}$ IPTG, and the cells were allowed to grow at $18{ }^{\circ} \mathrm{C}$ for $20 \mathrm{~h}$. The cells were harvested by centrifugation at $4000 \times g$ for $20 \mathrm{~min}$, resuspended in lysis buffer (40 mM Tris- $\mathrm{HCl}, \mathrm{pH}$ 8.0), and disrupted by ultrasonication (Sonic Dismembrator Model 500, Fisher Scientific). The cell debris was removed by centrifugation at $13,000 \times g$ for $40 \mathrm{~min}$, and the supernatant was loaded onto Ni-NTA agarose (QIAGEN). The bound proteins were eluted with $300 \mathrm{mM}$ imidazole in lysis buffer. The purified protein was concentrated to $3.1 \times 10^{-4} \mathrm{mM}$ in $40 \mathrm{mM}$ Tris- $\mathrm{HCl} \mathrm{pH} \mathrm{8.0.}$

Thioesterase activity was measured on a UV-1800 UV-Vis Spectrophotometer (Shimadzu, Japan). The reaction mixture contained $50 \mathrm{mM}$ of potassium phosphate buffer (pH 7.0), $80 \mu \mathrm{g} / \mathrm{mL}$ of BSA, $0.1 \mathrm{mM}$ of DTNB [5,5'-dithiobis-(2-nitrobenzoic acid)], $3.1 \times 10^{-5} \mathrm{mM}$ of enzyme, and a substrate $(0,2,4,6,8,12,16,20$, or $24 \mu \mathrm{M}$ palmitoyl-CoA) in the volume of $1 \mathrm{~mL}$ [31]. The reduction of DTNB by released CoA was monitored at $412 \mathrm{~nm}$ for 1-3 min. The kinetic parameters were calculated by nonlinear repression plots of the Michaelis-Menten equation. 


\section{Additional files}

Additional file 1. Additional figures.

Additional file 2. Additional tables.

\begin{abstract}
Abbreviations
ACC: acetyl-CoA carboxylase in E. coli; ACP: acyl carrier protein; acyl-CoA: acyl coenzyme A; FabB: $\beta$-ketoacyl-ACP synthase I in E. coli; FabF: $\beta$-ketoacyl-ACP synthase II in E. coli; FabH: $\beta$-ketoacyl-ACP synthase III in E. coli; Fabl: enoyl-ACP reductase in E. coli; FACS: fluorescence-activated cell sorting; FadD: acyl-CoA ligase in E. coli; FadR: multifunctional dual regulator of fatty acid metabolism in E. coli; FFA: free fatty acid; IPTG: isopropyl 1-thio- $\beta$-D-galactopyranoside; MC: Michaelis complex; $\mathrm{OD}_{600}$ : optical density at wavelength of $600 \mathrm{~nm}$; RFP: red fluorescent protein; 'TesA: leader sequence deleted thioesterase I from E. coli; TetA: tetracycline resistance protein.
\end{abstract}

\section{Authors' contributions}

KSS designed and conducted most of the experiments, analyzed the results, and wrote the manuscript. SK carried out protein purification and analysis of kinetic data. SKL designed the experiments, analyzed the data, provided the useful suggestions for experiments, and wrote the manuscript. All authors read and approved the final manuscript.

\section{Author details}

${ }^{1}$ School of Life Sciences, Ulsan National Institute of Science and Technology (UNIST), Ulsan 44919, Republic of Korea. ${ }^{2}$ School of Energy and Chemical Engineering, Ulsan National Institute of Science and Technology (UNIST), Ulsan 44919, Republic of Korea.

\section{Acknowledgements}

We are grateful to Dr. F. Zhang for comments on FFA analysis and fatty acid biosensor.

\section{Competing interests}

The authors declare that they have no competing interests.

\section{Availability of supporting data}

Supporting figures and tables are included within the article and its additional file.

\section{Funding}

This work was supported by the Next-Generation BioGreen 21 Program funded by the Ministry of Agriculture, Food and Rural Affairs (SSAC, Grant\#: PJ011181), and the Basic Science Research Program through the National Research Foundation of Korea (NRF) funded by the Ministry of Education (2013R1A1A2058667).

Received: 5 July 2016 Accepted: 24 September 2016

Published online: 06 October 2016

\section{References}

1. Rupilius W, Ahmad S. The changing world of oleochemicals. Palm Oil Dev. 2006:44:15-28.

2. Janssen HJ, Steinbuchel A. Fatty acid synthesis in Escherichia coli and its applications towards the production of fatty acid based biofuels. Biotechnol Biofuels. 2014;7:7.

3. Desbois AP, Smith VJ. Antibacterial free fatty acids: activities, mechanisms of action and biotechnological potential. Appl Microbiol Biotechnol. 2010;85:1629-42.

4. Jones AM, Klun JA, Cantrell CL, Ragone D, Chauhan KR, Brown PN, Murch SJ. Isolation and identification of mosquito (Aedes aegypti) biting deterrent fatty acids from male inflorescences of breadfruit (Artocarpus altilis (Parkinson) Fosberg). J Agric Food Chem. 2012;60:3867-73.
5. Hill J, Nelson E, Tilman D, Polasky S, Tiffany D. Environmental, economic, and energetic costs and benefits of biodiesel and ethanol biofuels. Proc Natl Acad Sci USA. 2006;103:11206-10.

6. Steen EJ, Kang Y, Bokinsky G, Hu Z, Schirmer A, McClure A, Del Cardayre SB, Keasling JD. Microbial production of fatty-acid-derived fuels and chemicals from plant biomass. Nature. 2010;463:559-62.

7. Yu AQ, Pratomo Juwono NK, Leong SS, Chang MW. Production of fatty acid-derived valuable chemicals in synthetic microbes. Front Bioeng Biotechnol. 2014:2:78.

8. Pfleger BF, Gossing M, Nielsen J. Metabolic engineering strategies for microbial synthesis of oleochemicals. Metab Eng. 2015;29:1-11.

9. Sathesh-Prabu C, Lee SK. Production of long-chain alpha, omega-dicarboxylic acids by engineered Escherichia coli from renewable fatty acids and plant oils. J Agric Food Chem. 2015;63:8199-208.

10. Kim SM, Choi BY, Ryu YS, Jung SH, Park JM, Kim GH, Lee SK. Simultaneous utilization of glucose and xylose via novel mechanisms in engineered Escherichia coli. Metab Eng. 2015;30:141-8.

11. Zhang F, Carothers JM, Keasling JD. Design of a dynamic sensor-regulator system for production of chemicals and fuels derived from fatty acids. Nat Biotechnol. 2012:30:354-9.

12. Zhang F, Ouellet M, Batth TS, Adams PD, Petzold CJ, Mukhopadhyay A, Keasling JD. Enhancing fatty acid production by the expression of the regulatory transcription factor FadR. Metab Eng. 2012;14:653-60.

13. Xu P, Gu Q, Wang W, Wong L, Bower AG, Collins CH, Koffas MA. Modular optimization of multi-gene pathways for fatty acids production in E. coli. Nat Commun. 2013:4:1409.

14. Xu P, Li L, Zhang F, Stephanopoulos G, Koffas M. Improving fatty acids production by engineering dynamic pathway regulation and metabolic control. Proc Natl Acad Sci USA. 2014;111:11299-304.

15. Lo YC, Lin SC, Shaw JF, Liaw YC. Crystal structure of Escherichia coli thioesterase I/protease I/lysophospholipase L1: consensus sequence blocks constitute the catalytic center of SGNH-hydrolases through a conserved hydrogen bond network. J Mol Biol. 2003;330:539-51.

16. Lee LC, Lee YL, Leu RJ, Shaw JF. Functional role of catalytic triad and oxyanion hole-forming residues on enzyme activity of Escherichia coli thioesterase I/protease I/phospholipase L1. Biochem J. 2006:397:69-76

17. Lu X, Vora H, Khosla C. Overproduction of free fatty acids in E. coli: implications for biodiesel production. Metab Eng. 2008;10:333-9.

18. Yu X, Liu T, Zhu F, Khosla C. In vitro reconstitution and steady-state analysis of the fatty acid synthase from Escherichia coli. Proc Natl Acad Sci USA. 2011;108:18643-8.

19. Dueber JE, Wu GC, Malmirchegini GR, Moon TS, Petzold CJ, Ullal AV, Prather KL, Keasling JD. Synthetic protein scaffolds provide modular control over metabolic flux. Nat Biotechnol. 2009;27:753-9.

20. Jiang P, Cronan JE Jr. Inhibition of fatty acid synthesis in Escherichia coli in the absence of phospholipid synthesis and release of inhibition by thioesterase action. J Bacteriol. 1994;176:2814-21.

21. Xu P, Wang W, Li L, Bhan N, Zhang F, Koffas MA. Design and kinetic analysis of a hybrid promoter-regulator system for malonyl-CoA sensing in Escherichia coli. ACS Chem Biol. 2014;9:451-8.

22. Rogers JK, Church GM. Genetically encoded sensors enable realtime observation of metabolite production. Proc Natl Acad Sci USA. 2016;113:2388-93.

23. Iram SH, Cronan JE. Unexpected functional diversity among FadR fatty acid transcriptional regulatory proteins. J Biol Chem. 2005;280:32148-56.

24. Lo YC, Lin SC, Shaw JF, Liaw YC. Substrate specificities of Escherichia coli thioesterase I/protease I/lysophospholipase L1 are governed by its switch loop movement. Biochemistry. 2005;44:1971-9.

25. Collins $\mathrm{CH}$, Leadbetter JR, Arnold FH. Dual selection enhances the signaling specificity of a variant of the quorum-sensing transcriptional activator LuxR. Nat Biotechnol. 2006;24:708-12.

26. Ran N, Frost JW. Directed evolution of 2-keto-3-deoxy-6-phosphogalactonate aldolase to replace 3-deoxy-D-arabino-heptulosonic acid 7-phosphate synthase. J Am Chem Soc. 2007:129:6130-9.

27. Yang JS, Seo SW, Jang S, Jung GY, Kim S. Rational engineering of enzyme allosteric regulation through sequence evolution analysis. PLoS Comput Biol. 2012;8:e1002612. 
28. Huang YT, Liaw YC, Gorbatyuk VY, Huang TH. Backbone dynamics of Escherichia coli thioesterase/protease l: evidence of a flexible active-site environment for a serine protease. J Mol Biol. 2001;307:1075-90.

29. Lee LC, Chou YL, Chen HH, Lee YL, Shaw JF. Functional role of a nonactive site residue $\operatorname{Trp}(23)$ on the enzyme activity of Escherichia coli thioesterase I/protease I/lysophospholipase L(1). Biochim Biophys Acta. 2009;1794:1467-73.

30. Choi YJ, Lee SY. Microbial production of short-chain alkanes. Nature. 2013;502:571-4

31. Tyukhtenko SI, Litvinchuk AV, Chang CF, Lo YC, Lee SJ, Shaw JF, Liaw YC, Huang TH. Sequential structural changes of Escherichia coli thioesterase/ protease I in the serial formation of Michaelis and tetrahedral complexes with diethyl p-nitrophenyl phosphate. Biochemistry. 2003;42:8289-97.

32. Davis MS, Cronan JE Jr. Inhibition of Escherichia coli acetyl coenzyme A carboxylase by acyl-acyl carrier protein. J Bacteriol. 2001;183:1499-503.

33. Heath RJ, Rock CO. Regulation of fatty acid elongation and initiation by acyl-acyl carrier protein in Escherichia coli. J Biol Chem. 1996;271:1833-6.

34. Heath RJ, Rock CO. Regulation of malonyl-CoA metabolism by acyl-acyl carrier protein and beta-ketoacyl-acyl carrier protein synthases in Escherichia coli. J Biol Chem. 1995;270:15531-8.

35. Bergler H, Fuchsbichler S, Hogenauer G, Turnowsky F. The enoyl-[acylcarrier-protein] reductase (Fabl) of Escherichia coli, which catalyzes a key regulatory step in fatty acid biosynthesis, accepts $\mathrm{NADH}$ and $\mathrm{NADPH}$ as cofactors and is inhibited by palmitoyl-CoA. Eur J Biochem. 1996;242:689-94.
36. Datsenko KA, Wanner BL. One-step inactivation of chromosomal genes in Escherichia coli K-12 using PCR products. Proc Natl Acad Sci USA. 2000;97:6640-5.

37. Muranaka N, Sharma V, Nomura Y, Yokobayashi Y. An efficient platform for genetic selection and screening of gene switches in Escherichia coli. Nucleic Acids Res. 2009;37:e39.

38. Warrens AN, Jones MD, Lechler RI. Splicing by overlap extension by PCR using asymmetric amplification: an improved technique for the generation of hybrid proteins of immunological interest. Gene. 1997;186:29-35.

39. Zheng L, Baumann U, Reymond JL. An efficient one-step sitedirected and site-saturation mutagenesis protocol. Nucleic Acids Res. 2004;32:e115

40. Blattner FR, Plunkett G 3rd, Bloch CA, Perna NT, Burland V, Riley M, Collado-Vides J, Glasner JD, Rode CK, Mayhew GF, et al. The complete genome sequence of Escherichia coli K-12. Science. 1997;277:1453-62.

41. Studier FW, Moffatt BA. Use of bacteriophage T7 RNA polymerase to direct selective high-level expression of cloned genes. J Mol Biol. 1986;189:113-30.

42. Lee TS, Krupa RA, Zhang F, Hajimorad M, Holtz WJ, Prasad N, Lee SK, Keasling JD. BglBrick vectors and datasheets: a synthetic biology platform for gene expression. J Biol Eng. 2011;5:12.

43. Kovach ME, Elzer PH, Hill DS, Robertson GT, Farris MA, Roop RM 2nd, Peterson KM. Four new derivatives of the broad-host-range cloning vector pBBR1MCS, carrying different antibiotic-resistance cassettes. Gene. 1995;166:175-6.

\section{Submit your next manuscript to BioMed Central and we will help you at every step:}

- We accept pre-submission inquiries

- Our selector tool helps you to find the most relevant journal

- We provide round the clock customer support

- Convenient online submission

- Thorough peer review

- Inclusion in PubMed and all major indexing services

- Maximum visibility for your research

Submit your manuscript at www.biomedcentral.com/submit 